Received: 22 May 2018

Accepted: 24 August 2018

Published online: 11 September 2018

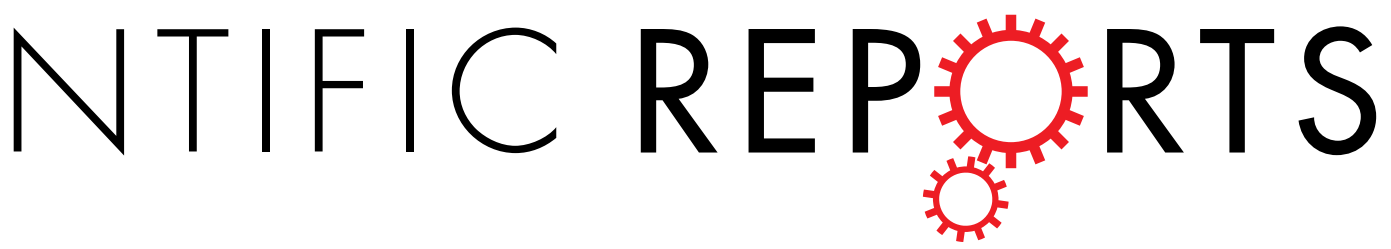

\title{
OPEN A flexible high speed pulse chopper system for an inverted neutron time-of-flight option on backscattering spectrometers
}

Markus Appel $\mathbb{C}^{1,2}$, Bernhard Frick $\mathbb{C}^{1}$ \& Andreas Mager ${ }^{2}$

We present the design and simulation of a high resolution inverted time-of-flight option for a neutron spectrometer with crystal analysers in backscattering, with specific reference to the IN16B spectrometer at the Institut Laue-Langevin, Grenoble. While the conventional configuration with Si 111 crystals provides sub- $\mu \mathrm{eV}$ resolution in an energy range limited to $\pm 30 \mu \mathrm{eV}$, the novel BATS option (BATS: Backscattering and Time-of-flight Spectrometer) extends the energy window to $340 \mu \mathrm{eV}$ with only a slightly increased resolution of $1.2 \mu \mathrm{eV}$. Moreover, the observation window can be shifted to inelastic energy transfers. To bring this about, a novel fast chopper system with disks of large diameter and complex slit pattern is used, offering high flexibility in resolution and repetition rate. The chopper system consists out of two counter rotating disk chopper pairs. It provides 7 different pulse lengths, three pulse repetition rates up to $237 \mathrm{~Hz}$ and can operate with Si 111 or Si 311 crystal analysers. The latter option is a unique feature which covers a Q-range up to $3.7 \AA^{-1}$ with a resolution of $6.8 \mu \mathrm{eV}$. Extensive ray-tracing simulations have been used to validate the design of the pulse chopper system, set limits on the sample size, and assess the achievable energy resolutions of the different chopper configurations.

Neutron spectrometers for inelastic or quasielastic scattering experiments use different techniques to reach a given energy resolution and access a specific region of the momentum vs. energy transfer space $(Q, \omega)$. Direct time-of-flight (TOF) spectrometers feature a monochromatic short pulse incident beam and a limited flight path in the secondary spectrometer. As a consequence, slow long wavelength neutrons, of up to $16 \AA$, are needed to achieve energy resolutions down to several $\mu \mathrm{eV}$, which in turn limits the accessible range of elastic momentum transfer Q. Neutron backscattering (BS) spectrometers use Bragg angles of $90^{\circ}$ on a crystal monochromator and analyser to achieve much higher sub- $\mu \mathrm{eV}$ resolution at shorter wavelengths, typically e.g. $6.27 \AA$ for the Si 111 reflection, but at the cost of a restricted accessible energy transfer window. The gap between these two techniques is bridged by inverted TOF-BS spectrometers ${ }^{1-6}$ by taking advantage of the strong points of the former two techniques. Present designs have a long flight path in the primary spectrometer with a quasi-white, short pulsed incident beam and use a near-backscattering crystal analyser in the secondary spectrometer.

Commonly, inverted TOF spectrometers are installed at pulsed neutron sources, exploiting the high peak intensity concentrated in short bursts of the source. The BATS option (BATS: Backscattering and Time-of-flight Spectrometer) implements this concept at a continuous source and is a novelty pursued mainly for two reasons: Firstly, it allows to extend substantially the energy range of a reactor-based backscattering spectrometer and thus the experimental possibilities. Secondly, a continuous source offers more flexibility in that the pulse repetition rate of the spectrometer is not bound to the source frequency ranging typically between $14 \mathrm{~Hz}$ and $60 \mathrm{~Hz}$ for spallation neutron sources, whereas the BATS option described in this paper allows for a repetition rate of up to $237 \mathrm{~Hz}$.

A first study for the BATS option was published by van Eijck et al. ${ }^{7}$ and its implementation was foreseen in the original design of the backscattering instrument IN16B at the Institute Laue-Langevin in Grenoble (France) which was commissioned in 2013 and has since served the user community as a powerful, conventional high

${ }^{1}$ Institut Laue-Langevin, 71 Avenue des Martyrs, 38000, Grenoble, France. ${ }^{2}$ Center for Medical Physics and Technology, Friedrich-Alexander Universität Erlangen-Nürnberg, Henkestrasse 91, 91052, Erlangen, Germany. Correspondence and requests for materials should be addressed to M.A. (email: appel@ill.eu) 


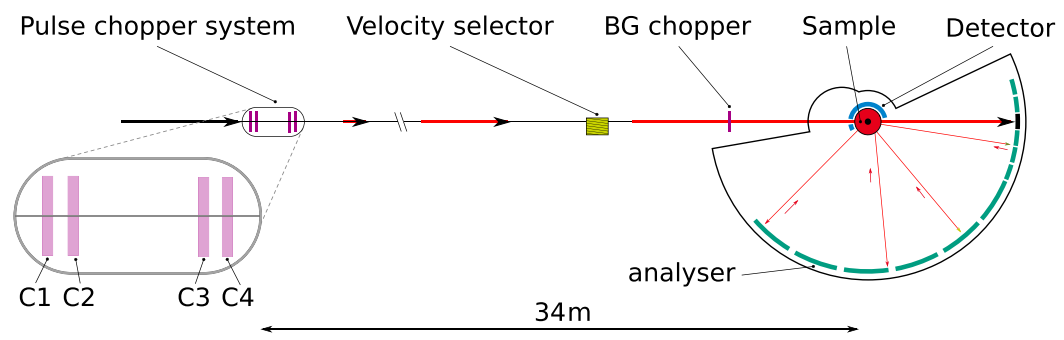

Figure 1. Scheme of the IN16B spectrometer in BATS configuration.

(a)

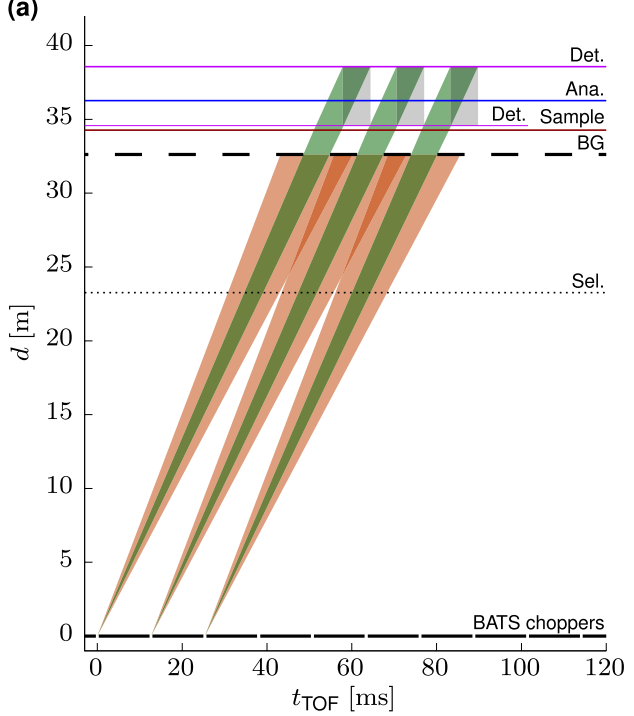

(b)

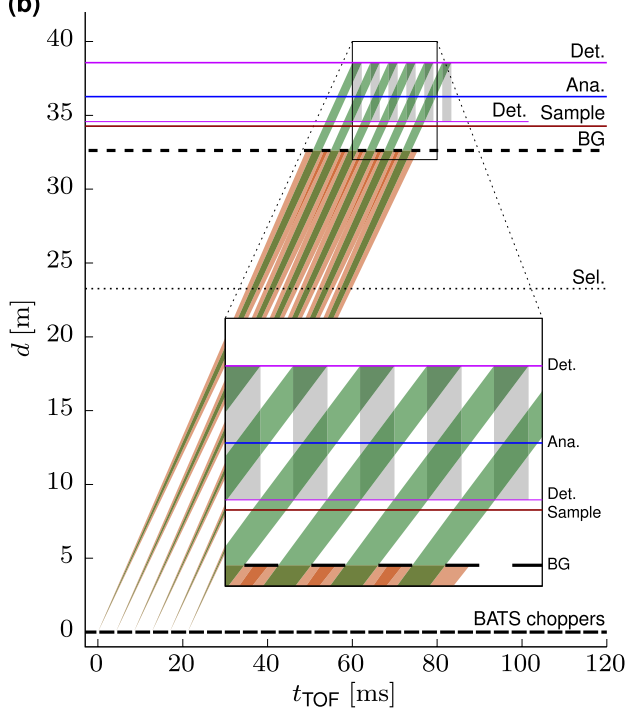

Figure 2. Distance vs. time diagrams of neutron pulses in the BATS configuration for the (a) Si 111 LRR mode and (b) Si 111 HRR mode.

resolution spectrometer at a reactor source ${ }^{8}$. In the following, we will extend the basic concept reported for BATS in ref. ${ }^{7}$ and present the details of a much more versatile chopper system with a variable energy resolution extending down to $1.2 \mu \mathrm{eV}$. The resulting design is supported by extensive ray-tracing simulations and is the base for a flexible 4-disk chopper system that was developed, built and installed over the last years. Hot commissioning of BATS is scheduled for spring 2018.

Inverted TOF Mode and Requirements for the BATS Chopper System

While the incident neutron energy in a conventional backscattering spectrometer is defined and modulated by a moving crystal monochromator on a Doppler drive, in BATS mode, it is determined by the different flight times of neutrons propagating from a short, quasi-white neutron pulse created by the chopper system. In both cases, the energy of neutrons scattered from the sample is analysed with crystal analysers. Figure 1 shows the IN16B BATS configuration with the beam structure created at the pulse chopper system, propagating through the wavelength band limiting neutron velocity selector to the background (BG) chopper and finally into the secondary spectrometer.

The analyser of backscattering spectrometers like IN16B have spherical geometry with the sample in the centre to attain best energy resolution. As a consequence, the incoming neutron beam must be pulsed with a duty cycle of at most $50 \%$ to distinguish neutrons scattered into the detector with and without energy analysis at the backscattering crystals. The possible pulse repetition rates are related to the distance between sample and analyser of $d_{\mathrm{SA}}=2 \mathrm{~m}$. With Si 111 analysers, the wavelength is $\lambda_{0}=6.271 \AA$ corresponding to a neutron velocity of $v_{0}=630.8 \mathrm{~ms}^{-1}$. This yields a flight time from sample to analyser and back of $t_{\mathrm{SAS}}=6.34 \mathrm{~ms}$, which defines the maximum length of the neutron pulses at $50 \%$ duty cycle. The corresponding repetition rate is thus $f_{0}=\left(2 t_{\mathrm{SAS}}\right)^{-1}=79 \mathrm{~Hz}$. Figure $2 \mathrm{a}$ depicts this pulse structure in a time vs. distance plot. Short pulses with frequency $f_{0}$ are created by the chopper system, the wavelength spectrum being restricted by the following velocity selector to a distribution with $\Delta \lambda / \lambda \approx 12 \%$ full width half maximum (FWHM). The BG chopper operates at the same frequency $f_{0}$ and transmits the central part of the spread pulse onto the sample (green), blocking out the overlapping tails of subsequent pulses (red). Note that the detector appears twice in this representation to indicate the two possible flight paths 'Sample-Detector' and 'Sample-Analyser-Detector', respectively. The time periods when energy analysed neutrons arrive on the detector are indicated by grey boxes. The width of the 


\begin{tabular}{|l|l|l|l|l|l|l|}
\hline Instrument & $\boldsymbol{d}[\mathbf{m m}]$ & $\boldsymbol{f}[\mathbf{r p m}]$ & $\boldsymbol{v}[\mathbf{m} / \mathbf{s}]$ & $\boldsymbol{a}\left[\mathbf{1 0}^{\mathbf{6}} \mathbf{m} / \mathbf{s}^{2}\right]$ & Material & Ref. \\
\hline BATS & 750 & 19000 & 746 & 1.48 & oCFRP & \\
\hline TOFTOF & 600 & 22000 & 691 & 1.59 & CFRP & 15 \\
\hline IN5 & 750 & 17000 & 668 & 1.19 & Al & 16 \\
\hline DCS & 580 & 20000 & 607 & 1.27 & Al & 17 \\
\hline LET & 633 & 18000 & 597 & 1.12 & CFRP & 18,19 \\
\hline
\end{tabular}

Table 1. Properties of fast turning disk choppers of selected neutron spectrometers; disk diameter $d$, rotation frequency $f$, circumferential disk speed $v$, circumferential radial acceleration $a$, disk material.

incident wavelength band is determined by the distance of the BATS choppers from the sample and is ideally matched to the wavelength band transmitted by the velocity selector. This results in a chopper to sample distance of $d_{\mathrm{CS}}=d_{\mathrm{SAS}} \times(\Delta v / v)^{-1}=33.3 \mathrm{~m}$, noting that $\Delta v / v=\Delta \lambda / \lambda$.

Additionally, the secondary spectrometer allows for operation in a 'high repetition rate' mode (HRR) at $3 f_{0}=237 \mathrm{~Hz}$ at the expense of a reduction in the energy range as shown in Fig. 2b. Here, the analysed neutrons from each pulse are counted while the subsequent pulse has already scattered on the sample and is being reflected from the analyser. This mode allows for a threefold increase of the spectral neutron intensity on the sample due to the increased pulse frequency, but requires restriction of the wavelength band to one third yielding $\Delta \lambda / \lambda=4 \%$ to avoid pulse overlap. As the width of the spectrum transmitted by the velocity selector cannot be changed easily, additional bandwidth choppers must be used.

The good energy resolution in a conventional BS spectrometer is mainly due to a well defined $\Delta d / d$ of the perfect crystal monochromator and the analysers in backscattering ${ }^{9}$. For the TOF-BS spectrometer BATS the secondary spectrometer comprising the analysers is identical, while the resolution of the primary spectrometer is dominated by the length of the neutron pulse created by the choppers. Ideally, the contributions of primary and secondary spectrometer are matched, which, in case of BATS, turns out to be the most critical requirement for the pulse chopper system. With an energy resolution of the secondary spectrometer with standard Si 111 analysers of $\delta E=0.6 \mu \mathrm{eV}$ at a neutron energy of $E_{0}=2.08 \mathrm{meV}$, pulses as short as $\delta t=1 / 2 \times \delta E / E \times d_{\mathrm{CS}} / v_{0}=7.6 \mu$ s are needed for a matched spectrometer. As the intensity is decreasing for shorter pulses cut from a continuous source, the chopper system should provide a reasonable set of pulse lengths to choose from. This allows to trade off intensity vs. resolution depending on the needs of the experiment to be performed.

Further, the BATS chopper system should allow for the use of Si 311 analysers in the secondary spectrometer which work with a wavelength of $3.27 \AA$ and allow to nearly double the maximum accessible $Q$ to $3.7 \AA^{-1}$. The higher velocity of these neutrons yields a repetition rate of $f_{0}^{\mathrm{Si} 311}=151 \mathrm{~Hz}$ with a pulse structure similar to the 'low repetition rate' mode (LRR) shown in Fig. 2a.

\section{Pulse Chopper System Design}

Disk size, speed and slit widths. The existing neutron guide at the location of the chopper system is optimised for transporting a continuous beam for conventional backscattering with Si 111 or Si 311 crystals, i.e., for wavelength bands around $6.27 \AA$ and $3.27 \AA$ respectively ${ }^{10}$. It has thus a large, rectangular cross section of $90 \mathrm{~mm}$ width and $115 \mathrm{~mm}$ height with a reflective coating of $m=2$, meaning that the critical angle of reflection of the walls is twice the critical angle of a simple Ni coating.

Given such large guide dimensions the circumferential velocity of the chopper disks is the most crucial aspect to attain the short pulse lengths needed, implying that both the rotation frequency and diameter of the disks should be maximised. This can be achieved by rotating the pulse chopper with a higher frequency which is an integer multiple $n$ of the base frequency $f_{0}$ and then using an additional suppressor chopper to skip $n-1$ pulses. Moreover, a counter-rotating disk pair can be used to reduce the pulse length by a factor of two compared to a single disk. The diameter of the disks is subject to technical limitations, in particular since several large slits with different size are required to provide different pulse lengths for varying the energy resolution. With recent progress in the manufacturing technique of radially oriented carbon fibre reinforced polymer (oCFRP) disks ${ }^{11}$ it is now possible to make choppers of $750 \mathrm{~mm}$ diameter rotating at $4 f_{0}=315 \mathrm{~Hz}$ corresponding to a circumferential speed of $746 \mathrm{~m} \mathrm{~s}^{-1}$, higher than the speed of any chopper system currently in operation (see Table 1).

The angular opening of a slit providing full view of the guide is $20.5^{\circ}$. These slits create a neutron pulse length of $90 \mu \mathrm{s}$ FWHM in case of two counter-rotating disks at $315 \mathrm{~Hz}$, which is one order of magnitude longer than the $7.6 \mu \mathrm{s}$ estimated in the previous section to match the resolution of the secondary spectrometer. This can only be attained by reducing the slit width down to $2^{\circ}$, which leads to a corresponding high loss of intensity due to reduction of the used guide cross section by a factor of 8 . However, these losses can be mediated by adapting the neutron optics around the chopper system. The concept for an adaptive focusing of this large cross section guide to focus the neutron beam on narrower slits is discussed in ref. ${ }^{12}$.

In summary it is thus crucial that the BATS chopper system provides multiple slits of different widths between matched resolution $\left(2^{\circ}\right)$ and full guide view $\left(20.5^{\circ}\right)$ and includes a slower suppressor chopper to transmit only the base pulse frequency $f_{0}$. As an alternative to disk choppers, a Fermi chopper (i.e. a rotating collimator) could be used but was found to be less flexible and to perform suboptimal towards shorter pulse lengths (see Supplementary Materials).

Disk slit pattern and modes of operation. The BATS chopper system uses 4 disk choppers grouped in two counter-rotating pairs as shown in Fig. 1. They are located at $34.2 \mathrm{~m}$ and $33.3 \mathrm{~m}$ upstream of the sample, 
(a)

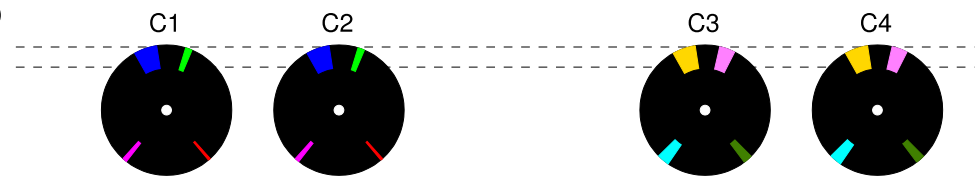

(b)

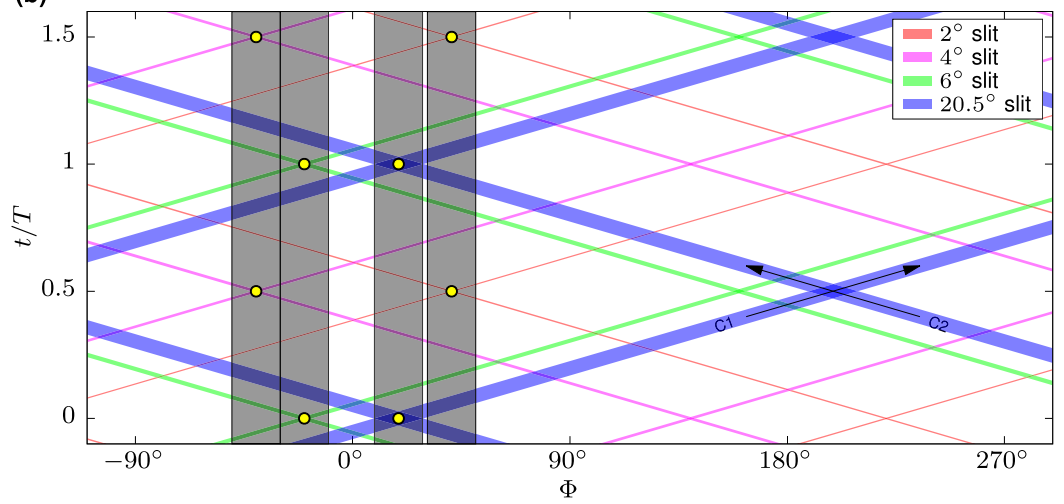

Figure 3. (a) Sketch of the slit pattern on the BATS disk chopper system; (b) Visualisation of overlapping slits during a full counter-rotation of the first chopper pair $\mathrm{C} 1 / \mathrm{C} 2$. Overlapping events of corresponding slit pairs are marked with circles, the four grey boxes correspond to the guide width centered at the respective markers.

respectively, where the exact positions needed to be adjusted due to constraints imposed by neighboring beamlines. Each disk features four slits of different width: $2^{\circ}, 4^{\circ}, 6^{\circ}, 20.5^{\circ}$ for the first disk pair, and $8^{\circ}, 11^{\circ}, 14^{\circ}, 20.5^{\circ}$ for the second pair. The $20.5^{\circ}$ opening corresponds to full guide view and is used to park the choppers in full transmission to recover the conventional backscattering mode of operation. The positions of the slits in the disks are sketched in Fig. 3a. The slit arrangement has been defined such that only one corresponding slit pair overlaps within the guide during a full rotation of each disk pair. The graph shown in Fig. $3 \mathrm{~b}$ visualises this arrangement, showing the polar angle of each slit vs. relative time per period for the first counter-rotating disk pair. Slits on the first disk are represented by bands from bottom left to top right, while slits on the second disk run from bottom right to top left. Overlaps of corresponding slits are marked with a yellow circle. The four vertical, shaded boxes represent the width of the guide aligned on each marker respectively. In practice this is achieved by tuning the chopper phases. All spurious overlaps of non-matching slits are located outside of the guide cross section for all configurations. This representation is very helpful as it provides graphical evidence for clean measurement configurations. However, it ignores the finite distance between the chopper disks, and the divergence and wavelength distribution of the neutron beam. To avoid any spurious transmission it is thus crucial that the chopper system is examined with ray tracing simulations as discussed in the next section.

The use of two counter-rotating disk pairs with multiple slits offers great experimental flexibility and allows to provide all required modes of operation, i.e., the use of Si 111 (both LRR and HRR) as well as Si 311 analysers with variable resolution. Table 2 gives an overview of these modes with the chopper related parameters in the columns to the right. For the LRR mode with Si 111 analysers, either chopper pair can be run at $315 \mathrm{~Hz}$ to create short pulses while the other pair acts as suppressor chopper at a ratio of 4:1. One can choose one out of seven different slits available across both pairs $\left(2^{\circ}, 4^{\circ}, 6^{\circ}, 8^{\circ}, 11^{\circ}, 14^{\circ}, 20.5^{\circ}\right)$ to create the neutron pulse, providing a graduated choice in the compromise between energy resolution and intensity.

The Si 111 HRR mode is realised by running both pairs at the same speed of $3 f_{0}=237 \mathrm{~Hz}$, creating a short pulse with the first pair and limiting the transmitted wavelength band with the second pair. The slit in the second chopper pair, located downstream at a distance of $0.9 \mathrm{~m}$, is chosen such that the transmitted wavelength band is narrow enough to avoid frame overlap. Therefore in this mode only three different pulse lengths can be used, but in consideration of the reduced energy transfer window only the highest resolution modes are considered relevant. For use with Si 311 analysers, the 'low repetition rate' modes are adapted with a slightly reduced pulse chopper frequency of $2 f_{0}^{\mathrm{Si} 311}=302 \mathrm{~Hz}$ and a suppressor chopper ratio of 2:1 $(151 \mathrm{~Hz})$. The higher neutron energy selected by the Si 311 reflection leads to a broadened energy resolution $\delta E$, but in turn opens access to higher scattering vectors up to $3.7 \AA^{-1}$.

\section{Simulation of Instrument Performance}

Having multiple slits on the BATS chopper disks brings about greater flexibility, but demands a thorough verification of the design to avoid any unexpected, spurious transmission. For this purpose ray tracing simulations are an indispensable tool to complement the above analytical considerations. Building on earlier simulations of the $\mathrm{H} 112$ guide and IN16B as a starting point ${ }^{7,10}$, a modular description of the instrument using the McStas simulation package ${ }^{13}$ was developed. These were used to verify the slit patterns as well as to study many aspects of the instrument performance like neutron flux and energy resolution of primary and secondary spectrometer. Moreover, a future optimisation of the neutron optics has been explored. The simulation code is released in the Supplemental Materials and also available in an online repository ${ }^{14}$ for future development. 


\begin{tabular}{|c|c|c|c|c|c|c|c|}
\hline & \multirow[b]{2}{*}{$\delta E[\mu \mathrm{eV}]$} & \multirow[b]{2}{*}{$\Delta E$ win. $[\mu \mathrm{eV}]$} & \multirow[b]{2}{*}{$Q\left[\AA^{-1}\right]$} & \multirow[b]{2}{*}{$w_{\mathrm{s}} \times h_{\mathrm{s}}\left[\mathrm{mm}^{2}\right]$} & \multirow[b]{2}{*}{$E_{\mathrm{f}}[\mathrm{meV}]$} & \multirow{2}{*}{\begin{tabular}{|l|}
$\mathrm{C} 1 / \mathrm{C} 2$ \\
$f$ (slit width) $[\mathrm{Hz}]$ \\
\end{tabular}} & \multirow{2}{*}{\begin{tabular}{|l|}
$\mathrm{C} 3 / \mathrm{C} 4$ \\
$f$ (slit width) $[\mathrm{Hz}]$
\end{tabular}} \\
\hline & & & & & & & \\
\hline \multirow{7}{*}{ Si 111 LRR } & 1.2 & 340 & 0.2 to 1.9 & $4 \times 10$ & 2.08 & $315\left(2^{\circ}\right)$ & $79\left(20.5^{\circ}\right)$ \\
\hline & 2.1 & 340 & 0.2 to 1.9 & $4 \times 20$ & 2.08 & $315\left(4^{\circ}\right)$ & $79\left(20.5^{\circ}\right)$ \\
\hline & 2.9 & 340 & 0.2 to 1.9 & $4 \times 40$ & 2.08 & $315\left(6^{\circ}\right)$ & $79\left(20.5^{\circ}\right)$ \\
\hline & 3.9 & 340 & 0.2 to 1.9 & $10 \times 40$ & 2.08 & $79\left(20.5^{\circ}\right)$ & $315\left(8^{\circ}\right)$ \\
\hline & 5.8 & 340 & 0.2 to 1.9 & $22 \times 40$ & 2.08 & $79\left(20.5^{\circ}\right)$ & $315\left(\mathbf{1 1}^{\circ}\right)$ \\
\hline & 7.6 & 340 & 0.2 to 1.9 & $22 \times 40$ & 2.08 & $79\left(20.5^{\circ}\right)$ & $315\left(14^{\circ}\right)$ \\
\hline & 8.6 & 340 & 0.2 to 1.9 & $22 \times 40$ & 2.08 & $315\left(\mathbf{2 0 . 5 ^ { \circ } )}\right.$ & $79\left(20.5^{\circ}\right)$ \\
\hline \multirow{3}{*}{ Si 111 HRR } & 1.4 & 120 & 0.2 to 1.9 & $4 \times 10$ & 2.08 & $237\left(2^{\circ}\right)$ & $237\left(11^{\circ}\right)$ \\
\hline & 2.7 & 120 & 0.2 to 1.9 & $4 \times 40$ & 2.08 & $237\left(4^{\circ}\right)$ & $237\left(11^{\circ}\right)$ \\
\hline & 3.6 & 120 & 0.2 to 1.9 & $10 \times 40$ & 2.08 & $237\left(6^{\circ}\right)$ & $237\left(8^{\circ}\right)$ \\
\hline \multirow{7}{*}{ Si 311} & 6.8 & 1100 & 0.7 to 3.7 & $4 \times 10$ & 7.63 & $302\left(2^{\circ}\right)$ & $151\left(20.5^{\circ}\right)$ \\
\hline & 14 & 1100 & 0.7 to 3.7 & $7 \times 40$ & 7.63 & $302\left(4^{\circ}\right)$ & $151\left(20.5^{\circ}\right)$ \\
\hline & 20 & 1100 & 0.7 to 3.7 & $14 \times 40$ & 7.63 & $302\left(6^{\circ}\right)$ & $151\left(20.5^{\circ}\right)$ \\
\hline & 28 & 1100 & 0.7 to 3.7 & $22 \times 40$ & 7.63 & $151\left(20.5^{\circ}\right)$ & $302\left(8^{\circ}\right)$ \\
\hline & 39 & 1100 & 0.7 to 3.7 & $22 \times 40$ & 7.63 & $151\left(20.5^{\circ}\right)$ & $302\left(11^{\circ}\right)$ \\
\hline & 53 & 1100 & 0.7 to 3.7 & $22 \times 40$ & 7.63 & $151\left(20.5^{\circ}\right)$ & $302\left(14^{\circ}\right)$ \\
\hline & 59 & 1100 & 0.7 to 3.7 & $22 \times 40$ & 7.63 & $302\left(\mathbf{2 0 . 5}^{\circ}\right)$ & $151\left(20.5^{\circ}\right)$ \\
\hline
\end{tabular}

Table 2. Overview of different modes of operation of the BATS configuration with expected energy resolution FWHM $\delta E$, full width of the accessible window in energy transfer $\Delta E$, accessible momentum transfer range $Q$, sensible choice of sample size with width $w_{\mathrm{s}}$ and height $h_{\mathrm{s}}$, analysed neutron energy $E_{\mathrm{f}}$, rotation frequency $f$ of first $(\mathrm{C} 1 / \mathrm{C} 2)$ and second $(\mathrm{C} 3 / \mathrm{C} 4)$ chopper pair and used slit width. The bold face indicates which slit is used to create the short neutron pulse.

Slit pattern. Each of the configurations listed in Table 2 is checked to transmit pulses as intended, i.e. only through the selected slit pairs. As an example, Fig. 4a shows the pulse structure before and after the second disk of the first chopper pair for the first Si 111 HRR mode of Table 2. From the four pulses transmitted by the first disk, only the red pulse corresponding to the $2^{\circ}$ slit is selected by the counter-rotating second disk. Some spurious transmission in the simulated wavelength band of $1 \AA$ to $12 \AA$ from the large $20.5^{\circ}$ slit (blue, see arrow) of the second disk is found.

The second graph in Fig. $4 \mathrm{~b}$ shows the pulse structure before and after the second counter-rotating chopper pair $\mathrm{C} 3 / \mathrm{C} 4$, which selects only a narrow part of the spectrum expanded with flight time. The spurious transmission from the first pair (see arrow) has moved towards larger $\left(t-t_{0}\right)$ as it contains only neutrons from the short wavelength end of the spectrum. These are suppressed at this point as the disks of the second pair are closed during the respective time window of their arrival. If longer wavelengths than the simulated $12 \AA$ were to be transmitted by the BATS choppers, they would be eliminated by the neutron velocity selector.

Figure $4 \mathrm{c}$ shows the same train of three consecutive pulses before and after the BG suppressor chopper, located $32.7 \mathrm{~m}$ downstream of the first chopper $\mathrm{C} 1$. The pulses have expanded even more, and the faster neutrons of each pulse overlap with the slower neutrons from the previous pulse. However, the overlap region falls into the time period which is suppressed by the BG chopper to attain the necessary $<50 \%$ duty cycle for the secondary spectrometer as mentioned above. In this way only clean, separated pulses reach the sample position.

Energy resolution and acceptable sample size. While the opening time of the pulse chopper discussed above is the most crucial feature determining the energy resolution of the spectrometer, there are more factors that need careful attention to ensure a realistic estimation. They all relate to the distribution of lengths of flight paths for neutron rays from the pulse chopper to the detector. The relation between the distribution of flight path length $\Delta d$ and energy resolution $\Delta E$ can be expressed as $\Delta E / E=2 \Delta d / d$, meaning that $8 \mathrm{~mm}$ difference in total flight path length contributes $1 \mu \mathrm{eV}$ in energy resolution for neutrons at $E=2.08 \mathrm{meV}$ with $d=34.2 \mathrm{~m}$. It is thus critical to assess the contributions to the flight path distribution according to the following list:

- Neutron guide of primary spectrometer: The large $m=2$ neutron guide transports neutrons of finite divergence. Thus the length of the flight path depends on the angle of the ray reflected at the guide walls. Moreover, possible future modifications of the guide for adapted focusing on smaller chopper slits and improved focusing on the sample can change this distribution and need to be taken into account.

- Sample geometry: It is evident that the standard sample sizes used on IN16B (flat slab of $30 \times 40 \mathrm{~mm}^{2}$ or hollow cylinder with $22 \mathrm{~mm}$ diameter) are too big to attain an energy resolution below $\approx 5 \mu \mathrm{eV}$ in TOF mode as they introduce large distributions of flight path lengths. Acceptable sample sizes are obtained from simulations.

- Secondary spectrometer: The secondary spectrometer contains spherical analyser segments that are usually aligned on a common sphere centered on the sample position. Scattered neutrons are focused back on the sample position and projected on the cylindrical detector arranged on the opposite side behind the sample (c.f. Fig. 1), which introduces large differences in the flight path (on IN16B up to $40 \mathrm{~mm}$ ). Vertically position 

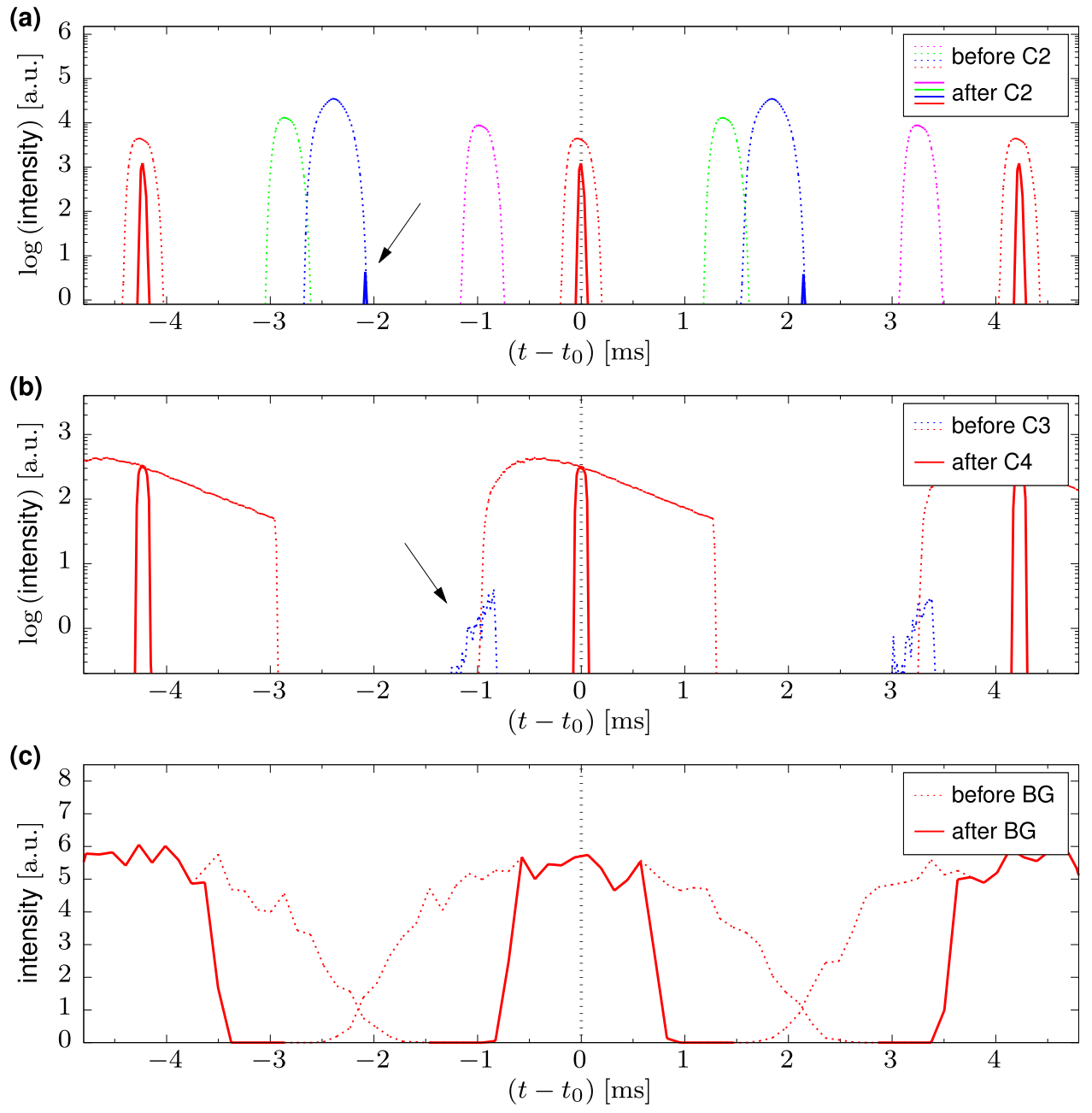

Figure 4. Simulated pulse transmission for the first Si 111 'high repetition rate' mode in Table 2 of (a) the C2 chopper, (b) the $\mathrm{C} 3 / \mathrm{C} 4$ chopper pair, and (c) the BG chopper ( $60^{\circ}$ opening). Curves are color coded according to the slit color of the first chopper $\mathrm{C} 1$ in Fig. 3. The horizontal axis shows the time relative to the time-of-flight $t_{0}$ of the central neutron wavelength at the respective instrument components. A spurious transmission of the first chopper pair is marked with the arrows; it is not transmitted by the second chopper pair.

sensitive detector tubes allow to correct for this contribution in data reduction, leaving only an uncertainty in the detection depth. On IN16B, this corresponds to a $13 \mathrm{~mm}$ thick ${ }^{3} \mathrm{He}$ volume, but due to its strong absorption the effective detection depth is much smaller, e.g. $50 \%$ of neutrons selected by $\mathrm{Si} 111$ analysers are absorbed within the first $1.5 \mathrm{~mm}$. This is also accounted for in the McStas simulations.

With a realistic modelling of all relevant effects, the ray tracing simulation can be expected to give a reliable estimation of the final energy resolution $\delta E$. In the simulation, the energy resolution is not obtained as in a real experiment by using a purely elastic scattering sample as this would be computationally inefficient. Instead, an artificial sample is used which scatters each neutron ray into a fixed energy window around the energy acceptance of the analyser, e.g. the range between $(2080 \pm 5) \mu \mathrm{eV}$ in case of Si 111 analysers. The energy resolution is then obtained by observing, as a function of energy transfer $\Delta E$, the difference $\left(\Delta E_{\text {meas }}-\Delta E_{\text {real }}\right)$ which refers to the following quantities: $\Delta E_{\text {meas }}$ is the energy transfer as it would be measured in the spectrometer, calculated from the time-of-flight of the neutron ray from the pulse chopper to the detection point; $\Delta E_{\text {real }}$ is the actual energy transfer applied to the neutron ray during the simulated scattering on the artificial sample. The difference of these two values thus corresponds to the resolution of the experimental setup. As an example, the resulting distribution for the first configuration in Table 2 is shown in Fig. 5. This simulation was run with the BG chopper stopped in order to observe the full wavelength band transmitted by the velocity selector. However, in reality, the BG chopper is indispensable and thus only a limited window of $\Delta E$ can be observed in the experiment. In light of envisaged future modifications of the neutron guide for adapted beam focusing, we simulate both the current guide as well as the focusing configuration from ref. ${ }^{12}$. We find slightly different values for the resolution in both cases, most probably because a larger phase space element of the incoming beam is picked up by the sample.

The simulated resolution function in the elastic region for some configurations using $\mathrm{Si} 111$ analysers is shown in Fig. 6a. The width of the resolution function depends on energy transfer as shown in Fig. 6b, narrowing for 


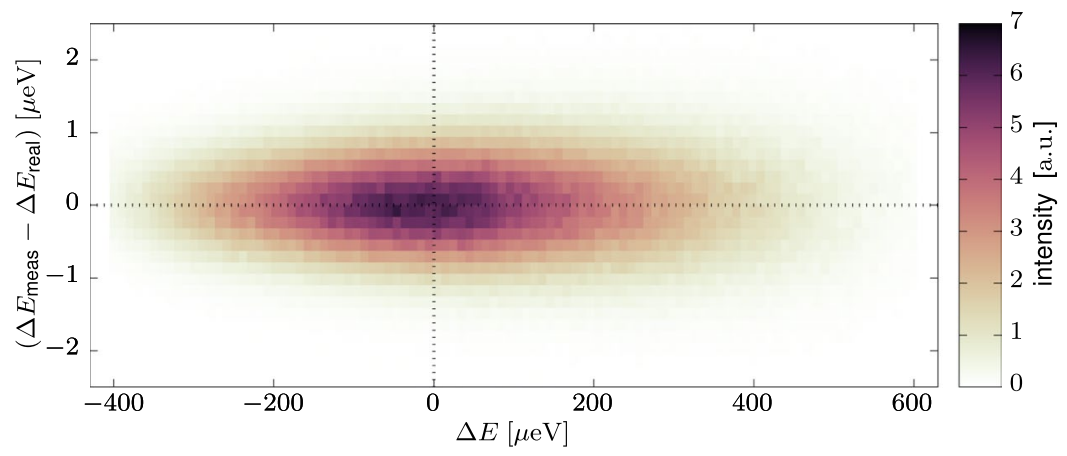

Figure 5. Simulated energy resolution function $\left(\Delta E_{\text {meas }}-\Delta E_{\text {real }}\right)$ vs. energy transfer $\Delta E$ for the highest resolution configuration (first line in Table 2).

(a)

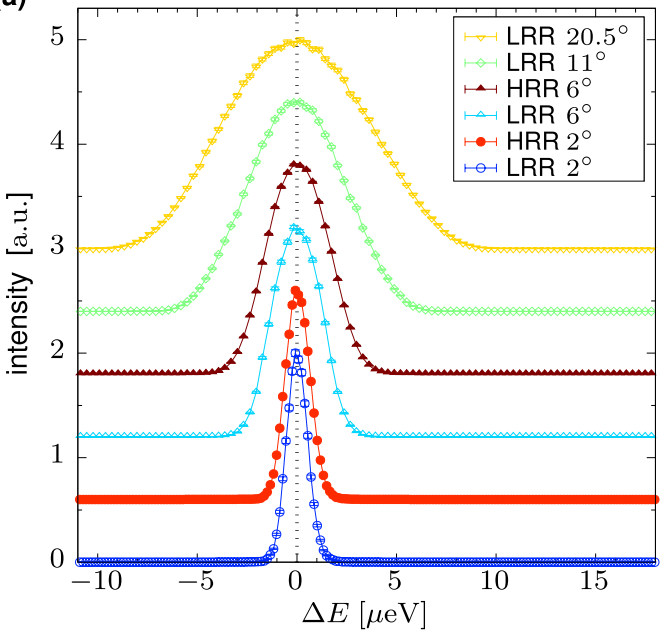

(b)

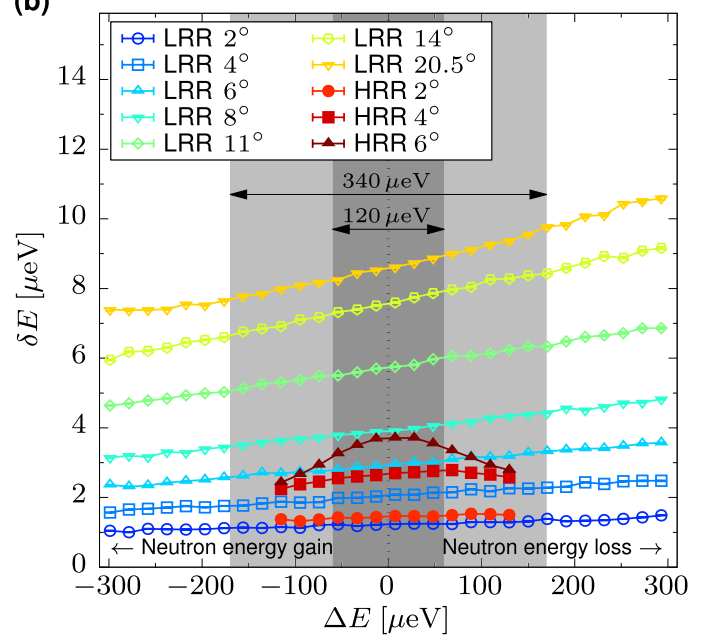

Figure 6. Simulated energy resolution function for Si 111 analysers with different chopper configurations of 'low repetition rate' (LRR) and 'high repetition rate' (HRR). The angle in the legend corresponds to the opening of the slit pair creating the pulse. (a) Elastic energy resolution function. The curves are offset vertically for clarity. (b) Dependence of the FWHM of the energy resolution width $\delta E$ on energy transfer $\Delta E$. The shaded areas visualize the accessible energy transfer window in case of elastic centering for LRR modes $(340 \mu \mathrm{eV})$ and HRR modes $(120 \mu \mathrm{eV})$.

$\Delta E<0$ as the incident energy $E_{i}$ is decreasing. A noticeable behavior is observed for the Si 111 HRR modes, where especially for the $6^{\circ}$ slit a strong triangular envelope appears on top of the weak monotonous dependency such that the resolution is narrowing both for $\Delta E<0$ and $\Delta E>0$. A similar, less pronounced effect can be seen at the extremities of the curve obtained for the $4^{\circ}$ slit. This is caused by the second chopper pair rotating at the same speed as the first pair and cutting off the wings of pulses for faster and slower neutrons, effectively narrowing the pulse width depending on neutron velocity. Note that the usable window for the HRR modes in $\Delta E$ is limited to a full width of at most $120 \mu \mathrm{eV}$, meaning that the enveloping effect will be noticeable only for the $6^{\circ}$ slit. Moreover, if the centre of the chosen energy transfer window is offset from $\Delta E=0$, the respective chopper phases shift the envelope function to follow the centre of such an inelastic window.

From a list of all elastic resolutions for different sample geometries (see Supplementary Tables S1 to S4), a reasonable limit for the sample size of each configuration can be chosen and it is reported together with the respective energy resolution in Table 2 . The simulated resolution with $\mathrm{Si} 111$ analysers ranges between $1.2 \mu \mathrm{eV}$ and $8.6 \mu \mathrm{eV}$ with sample sizes of $4 \times 10 \mathrm{~mm}^{2}$ for the best resolution up to the standard size of $22 \times 40 \mathrm{~mm}^{2}$ above $5 \mu \mathrm{eV}$. For Si 311 analysers we expect $6.8 \mu \mathrm{eV}$ to $59 \mu \mathrm{eV}$.

\section{Conclusion}

We report on the design of a flexible pulse chopper system for an inverted TOF option on a neutron backscattering spectrometer at a continuous neutron source with specific reference to the BATS option for the instrument IN16B at the Institute Laue-Langevin. In spite of a relatively short primary flight path and a wide neutron guide at the BATS chopper location, the expected high energy resolution is made possible by recent technological progress in chopper disk construction. Two counter rotating double disk choppers each with 4 slits of different width give variable modes of operation which offer unprecedented flexibility to the experimenter. For Si 111 analysers 
the energy resolution can be varied in seven steps between $1.2 \mu \mathrm{eV}$ and $8.6 \mu \mathrm{eV}$, 'high repetition rate' modes at $237 \mathrm{~Hz}$ provide increased flux at a reduced energy transfer range, and Si 311 analysers can be used giving access to momentum transfers $Q$ up to $3.7 \AA^{-1}$. The instrument performance was assessed by McStas ray tracing simulations, in particular to estimate the influence of sample size on the energy resolution. The simulations confirm that an inverted TOF backscattering spectrometer of the described BATS type will be most flexible and that its energy resolution will be highly competitive with any existing or planned TOF-BS spectrometer. As a significant advantage we note the possibility to realize much higher pulse repetition rates than available from spallation source frequencies. Future optimisations of the neutron optics are envisaged, allowing for adapted beam focusing on small slits of the chopper disks and improved focusing on the sample position.

\section{References}

1. Carlile, C. \& Adams, M. The design of the IRIS inelastic neutron spectrometer and improvements to its analysers. Physica B 182, 431-440 (1992).

2. Telling, M. T. F. \& Andersen, K. H. Spectroscopic characteristics of the OSIRIS near-backscattering crystal analyser spectrometer on the ISIS pulsed neutron source. Phys. Chem. Chem. Phys. 7, 1255-1261 (2005).

3. Telling, M. T. F., Campbell, S. I., Engberg, D., Martin y Marero, D. \& Andersen, K. H. Correction: Spectroscopic characteristics of the OSIRIS near-backscattering crystal analyser spectrometer on the ISIS pulsed neutron source. Phys. Chem. Chem. Phys. 18, 8243 (2016).

4. Mamontov, E. \& Herwig, K. W. A time-of-flight backscattering spectrometer at the Spallation Neutron Source, BASIS. Review of Scientific Instruments 82, 085109 (2011).

5. Shibata, K. et al. The performance of TOF near backscattering spectrometer DNA in MLF, J-PARC. JPS Conf. Proc. 8, 036022 (2015).

6. Tsapatsaris, N., Lechner, R. E., Markó, M. \& Bordallo, H. N. Conceptual design of the time-of-flight backscattering spectrometer, MIRACLES, at the European Spallation Source. Rev. Sci. Instrum. 87, 085118 (2016).

7. van Eijck, L., Gérard, L., Frick, B., Seydel, T. \& Schober, H. A case study for using neutron backscattering instruments at reactors in inverted time-of-flight mode. Nucl. Instrum. Methods Phys. Res., Sect. A672, 64-68 (2012)

8. Frick, B., Appel, M., Seydel, T., van Eijck, L. \& Bazzoli, D. Manuscript in preparation.

9. Frick, B. Neutron backscattering spectroscopy. In Hippert, F., Geissler, E., Hodeau, J., Lelièvre-Berna, E. \& Regnard, J.-R. (eds) Neutron and X-ray Spectroscopy, 483-527 (Springer Netherlands, 2006).

10. Bordallo, H. N., Frick, B., Schober, H. \& Seydel, T. Primary spectrometer neutron optics simulations for a new cold neutron backscattering spectrometer. J. Neutron Res. 16, 39-54 (2008).

11. Spiegelhalder, B., Keller, U. \& Käppeler, E. Chopperscheibe sowie Vorrichtung und Verfahren zu deren Herstellung German Patent No. DE 102014004994 B3 (2015)

12. Appel, M., Frick, B. \& Magerl, A. Manuscript in preparation.

13. Willendrup, P., Farhi, E. \& Lefmann, K. Mcstas 1.7-a new version of the flexible Monte Carlo neutron scattering package. Physica B 350, E735-E737 (2004).

14. Appel, M. Mcstas simulation code repository of the IN16B backscattering spectrometer. https://code.ill.fr/appel/in16b-mcstas. https://doi.org/10.5291/ILL-SOFTWARE.IN16B-MCSTAS.

15. Heinz Maier-Leibnitz, Z. et al. TOFTOF: Cold neutron time-of-flight spectrometer. J. large-scale Res. Facil. 1, A15 (2015).

16. Ollivier, J. \& Mutka, H. IN5 Cold neutron time-of-flight spectrometer, prepared to tackle single crystal spectroscopy. J. Phys. Soc. Jpn. 80, SB003 (2011).

17. The DCS Poster. Online, https://ncnr.nist.gov/instruments/dcs/dcs_post.html (2002).

18. Bewley, R., Taylor, J. \& Bennington, S. LET, a cold neutron multi-disk chopper spectrometer at ISIS. Nucl. Instrum. Methods Phys. Res., Sect. A637, 128-134 (2011).

19. Bewley, R. private comm. (2018).

\section{Acknowledgements}

The authors would like to thank Helmut Schober for his continuing support and helpful discussions. We acknowledge support by Deutsche Forschungsgemeinschaft and Friedrich-Alexander-Universität ErlangenNürnberg (FAU) within the funding programme Open Access Publishing. This work was supported by the German Ministry of Education and Research (BMBF) under grants number 05K13WE1 and 05K16WEA.

\section{Author Contributions}

M.A. conceived the chopper system design, carried out the simulations, analysed and prepared the results. B.F. devised BATS and initiated the project. A.M. coordinated the project and raised the funds. All authors participated in regular design reviews and the writing of the manuscript.

\section{Additional Information}

Supplementary information accompanies this paper at https://doi.org/10.1038/s41598-018-31774-y.

Competing Interests: The authors declare no competing interests.

Publisher's note: Springer Nature remains neutral with regard to jurisdictional claims in published maps and institutional affiliations.

Open Access This article is licensed under a Creative Commons Attribution 4.0 International License, which permits use, sharing, adaptation, distribution and reproduction in any medium or format, as long as you give appropriate credit to the original author(s) and the source, provide a link to the Creative Commons license, and indicate if changes were made. The images or other third party material in this article are included in the article's Creative Commons license, unless indicated otherwise in a credit line to the material. If material is not included in the article's Creative Commons license and your intended use is not permitted by statutory regulation or exceeds the permitted use, you will need to obtain permission directly from the copyright holder. To view a copy of this license, visit http://creativecommons.org/licenses/by/4.0/.

(C) The Author(s) 2018 